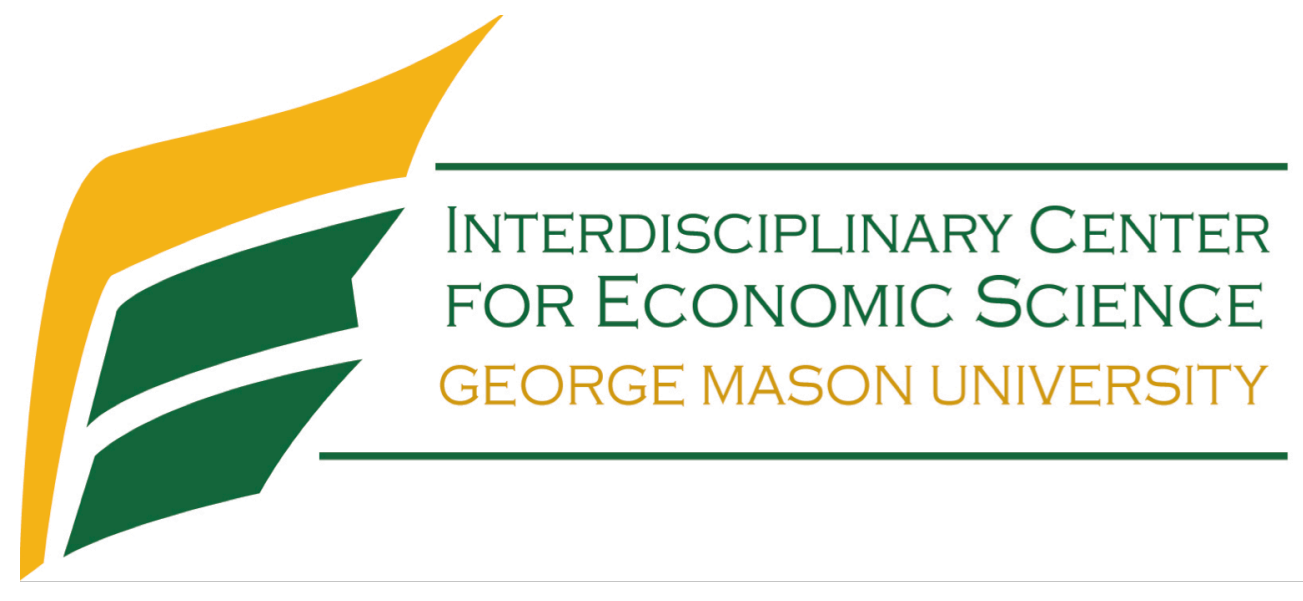

\title{
Electoral Accountability and Responsive Democracy
}

John Duggan and César Martinelli

October 2015

Discussion Paper

Interdisciplinary Center for Economic Science

4400 University Drive, MSN 1B2, Fairfax, VA 22030

Tel: +1-703-993-4719 Fax: +1-703-993-4851

ICES Website: http://ices.gmu.edu

ICES RePEc Archive Online at: http://edirc.repec.org/data/icgmuus.html 


\title{
Electoral Accountability and Responsive Democracy
}

\author{
John Duggan* César Martinelli ${ }^{\dagger}$
}

February 16, 2015

\begin{abstract}
We consider a canonical two-period model of elections with adverse selection (hidden preferences) and moral hazard (hidden actions), in which neither voters nor politicians can commit to future choices. We prove existence of electoral equilibria, and we show that office holders mix between "taking it easy" and "going for broke" in the first period. Even in the presence of a finite horizon, we establish that increasing office motivation leads to arbitrarily high expected policy outcomes. We conclude that the mechanism of electoral accountability has the potential to achieve responsiveness of democratic politics when electoral incentives are sufficiently large.
\end{abstract}

\section{Introduction}

Representative democracy, by definition, entails the delegation of power by society to elected officials. A main concern for representative democracy is then to devise means to discipline politicians in office to achieve desirable policy outcomes for citizens. Political thinkers since Madison, if not earlier, have considered the possibility of re-election to be an essential device in this regard, ${ }^{1}$ and the goal of this paper is to apply the tools of formal political theory to study the incentives provided by the mechanism of democratic elections and the implied linkage between voter preferences and policy outcomes. In doing so, we must move beyond the basic Downsian model of static elections, the stalwart of formal work on electoral

\footnotetext{
*Affiliation: Dept. of Political Science, University of Rochester. Email: dugg@ur.rochester.edu

${ }^{\dagger}$ Affiliation: Dept. of Economics, George Mason University. Email: cmarti33@gmu.edu

${ }^{1}$ The Federalist 57, in particular, offers a discussion of the role of "frequent elections" in the selection of politicians and the control of politicians while in office.
} 
competition, to explicitly incorporate a temporal dimension within the analysis. An active and growing literature on electoral accountability, starting with the seminal work of Barro (1973) and Ferejohn (1986), has undertaken this line of inquiry. The goal of this literature is to improve our understanding of the operation of realworld political systems and the conditions under which democracies succeed or fail. This, in turn, has the potential to facilitate the design of political institutions that produce socially desirable policy outcomes.

Nevertheless, our understanding of the fundamental interplay between disciplining incentives provided by the possibility of re-election and the incentives for opportunistic behavior in the present remains incomplete. With few exceptions, such as Fearon (1999), Ashworth (2005), and Ashworth and Bueno de Mesquita (2008), the literature on electoral accountability has paid relatively little attention to the situation in which the preferences and actions of politicians are unobserved by voters. Such settings combine salient aspects of real-world elections, but they are fraught with difficulties, beginning with the issue of the existence of equilibrium. As a consequence, research has been conducted under special modeling assumptions about the type space, the action space, or the information held by politicians. In this article, we present a formal model of elections that allows us to study the dynamic incentives of politicians, and the policy choices emerging from those incentives, in environments with realistically sparse information. We prove existence of equilibrium under relatively general conditions, and we give a characterization of equilibrium behavior in the model. In spite of the sparseness of information available to voters, we establish the possibility of responsive democracy when politicians are highly office motivated. That is, the incentives of re-election can induce politicians to exert high levels of effort, overcoming the divergence of interests between politicians and voters and mitigating the commitment problem posed by the electoral mechanism.

We conduct our research in the framework of a two-period model in which the incumbent politician in office in the first period faces a randomly chosen challenger in the second period. Variations of the two-period period model have been employed in the graduate textbooks of Persson and Tabellini (2000) and Besley (2006), providing a minimal setting to study intertemporal incentives; in this sense the two-period model can be regarded as canonical. We assume that politicians' preferences are private information, i.e., adverse selection is present, and that political choices are observed by voters only with some noise, i.e., they are subject to imperfect monitoring, or moral hazard. We consider the rent-seeking environment studied in the public choice tradition of Barro (1973) and Ferejohn (1986), in which politicians have a short-run incentive to shirk from effort while in office, or equivalently to engage in rent-seeking activities that hurt other citizens. Politicians differ with regard to their preference for rent-seeking (or equivalently, they differ 
in how much their cost of effort). We maintain the key assumption of the electoral accountability literature that neither politicians nor voters can commit to future actions, in the spirit of the citizen-candidate tradition of Osborne and Slivinski (1996) and Besley and Coate (1997). An implication is that in equilibrium, both the policy choices of politicians and the re-election standard used by voters must be time consistent, in the sense that first-period choices must be optimal in light of expected behavior in the future.

We develop a notion of electoral equilibrium, a refinement of perfect Bayesian equilibrium that imposes structure suitable to the formal study of elections in context of political science, namely, voters defer to the incumbent when indifferent, and voters are more willing to reelect incumbents after observed better policy outcomes. Put simply, electoral equilibrium requires that voters follow a straightforward retrospective rule: re-elect the incumbent if and only if the policy outcome is equal or better than some standard. We note that electoral equilibria must solve a non-trivial fixed point problem: the first period office holder's choice must take account of the re-election standard of voters, and the updating of voter beliefs depends on choices of the first period office holder via Bayes' rule. We impose sufficient structure (satisfied in special cases of interest) that a politician can have at most two optimal policy choices- "taking it easy" and "going for broke"and we use this to establish existence of electoral equilibrium. We then show that when politicians are highly office motivated, the re-election standard used by voters in equilibrium becomes arbitrarily demanding, and all "above average" politician types exert arbitrarily high effort in their first term of office. Although the increasing standard used by voters may lead some politician types to abandon the pursuit of re-election and shirk in the first term, this result implies that the expected effort level in the first period becomes arbitrarily high.

This responsive democracy result is superficially similar to the median voter theorem in the traditional Downsian framework, but the logic underlying it is very different: candidates cannot make binding campaign promises, and they do not compete for votes in the Downsian sense; rather, they are citizen candidates whose policy choices must maximize their payoffs in equilibrium, and the responsiveness result is driven by politicians' concern for reputation. Specifically, the desire to be re-elected can induce politicians to mimic types whose preferences are more closely aligned with voters, and if the reward for political office is large enough, then this incentive leads some types to exert arbitrarily high effort. Thus, electoral accountability engenders the possibility of responsive democracy, despite the paucity of instruments that the voters can yield, in contrast with the (static) principal-agent model in complete contract settings.

We emphasize that the equilibrium standard used by voters is optimal given politicians' choices, but it is not optimally set ex ante: voters do not set the stan- 
dard before the election in order to elicit maximal effort. Because voters, like politicians, face a commitment problem, they "best respond" in equilibrium by re-electing the incumbent when the expected payoff from doing so, conditional on the observed policy outcome, exceeds the prospects of a challenger; in other words, the equilibrium standard is time consistent. This facet of the equilibrium analysis stacks the deck against our responsive democracy result, and it means that responsiveness does not rely on any assumption that voters can commit ex ante to a socially optimal standard of re-election.

In Section 2, we present the two-period electoral accountability model. In Section 3, we define the electoral equilibria that are the focus of our analysis. In Section 4, we impose added structure on the model and take preliminary steps toward the main results, namely, showing the existence of at most two local maximizers to the problem faced by each type of politician in equilibrium. In Section 5 , we prove existence and provide a characterization of electoral equilibria, and we discuss the difficulties in achieving existence due to non-convexity of the first-term office holder's optimization problem. In Section 6, we present our result on responsive democracy as politicians become office motivated. In Section 7, we discuss in detail the relationship of our paper with the electoral accountability literature. In Section 8 , we gather final remarks.

\section{Electoral accountability model}

We analyze a two-period model of elections involving a representative voter, an incumbent politician, and a challenger. Prior to the game, nature chooses the types of the incumbent and challenger from the finite set $T=\{1, \ldots, n\}$, with $n \geqslant 2$. These types are private information and drawn identically and independently, and we let $p_{j}>0$ denote the prior probability that a politician is type $j$. In period 1 , the incumbent makes a policy choice $x_{1} \in X=\mathbb{R}_{+}$, which is unobserved by the voter, and a policy outcome $y_{1}$ is drawn from $Y=\mathbb{R}$ according to the distribution $F\left(\cdot \mid x_{1}\right)$. In contrast to the choice $x_{1}$, the outcome $y_{1}$ is observed by the voter. Then the voter chooses between the incumbent politician and the challenger. In period 2 , the winner of the election makes a policy choice $x_{2} \in X$, a policy outcome $y_{2}$ is drawn from $F\left(\cdot \mid x_{2}\right)$, and the game ends. Figure 1 illustrates the timeline of events in the model.

Given policy choice $x$ and outcome $y$ in either period, each player obtains a payoff of $u(y)$ if not in office, while an office holder of type $j$ receives a payoff of $w_{j}(x)+\beta$, where $\beta \geqslant 0$ represents the benefits of holding office. Total payoffs for the voter and politicians are the sum of per-period payoffs. The voter has increasing preferences over policy outcomes, while a politician who holds office incurs a cost 


$\begin{array}{cccccc}\text { Nature } & \text { Incumbent } & \text { Nature } & \text { Voters } & \text { Election } \\ \text { winner } & \text { Nature } \\ \begin{array}{c}\text { incumbent } \\ \begin{array}{c}\text { and challenger } \\ \text { types }\end{array}\end{array} & \begin{array}{c}\text { policy } \\ \text { choice } x_{1}\end{array} & \begin{array}{c}\text { policy } \\ \text { outcome } y_{1}\end{array} & \begin{array}{c}\text { re-elect } \\ \text { or not }\end{array} & \begin{array}{c}\text { policy } \\ \text { choice } x_{2}\end{array} & \begin{array}{c}\text { polime } \\ \text { outcome } y_{2}\end{array} \\ \end{array}$

Figure 1: Timeline

for higher policy choices. We assume that utilities have the simple form

$$
u_{j}(y)=u(y) \quad \text { and } \quad w_{j}(x)=\lambda_{j}\left(v(x)-\frac{1}{\theta_{j}} c(x)\right)+\kappa_{j}
$$

where $u: Y \rightarrow \mathbb{R}$ is continuous and strictly increasing, $v: X \rightarrow \mathbb{R}$ is differentiable, concave, and strictly increasing, $c: X \rightarrow \mathbb{R}_{+}$is continuously differentiable, strictly convex, and has positive derivative, and $\kappa_{j}, \lambda_{j}, \theta_{j}$ are type-dependent parameters satisfying $\lambda_{j}, \theta_{j}>0$ and $\theta_{1}<\theta_{2}<\cdots<\theta_{n}$. Thus, higher policy choices are more costly for higher politician types. We assume that if in office, each politician type has an optimal policy $\hat{x}_{j}$, and that at most the type 1 politicians have ideal policy equal to zero. Thus, our assumptions imply that the ideal policies of politicians are strictly ordered according to type:

$$
0 \leqslant \hat{x}_{1}<\hat{x}_{2}<\cdots<\hat{x}_{n}
$$

As we will see, the functional form for politicians' payoff is useful for establishing an ordering property of politicians' best responses to the voter's strategy. The functional form admits two simple specifications that are worthy of note. One common specification is quadratic utility, in which case $w_{j}(x)=-\left(x-\hat{x}_{j}\right)^{2}+K_{j}$, where $K_{j}$ is a constant. To obtain this, we set

$$
v(x)=2 x, c(x)=x^{2}, \kappa_{j}=-\hat{x}_{j}^{2}+K_{j}, \lambda_{j}=\theta_{j}=\hat{x}_{j} .
$$

Another specification of interest is is exponential utility, whereby $w_{j}(x)=-e^{x-\hat{x}_{j}}+$ $x+K_{j}$, which is obtained by setting

$$
v(x)=x, c(x)=e^{x}, \kappa_{j}=K_{j}, \lambda=1, \theta_{j}=e^{\hat{x}_{j}} .
$$

Note that we can assume politicians share the voter's preferences over policy outcomes by setting $\lambda_{j}=1$ and specifying that $v(x)$ is equal to the expected utility 
from policy outcomes generated by the choice $x$, i.e., $v(x)=\mathbb{E}[u(y) \mid x]$. In this case, an office holder differs from other citizens only by the cost term $\left(1 / \theta_{j}\right) c(x)$. In this version of the model, it is natural to view policy outcomes as a level of public good or (the inverse of) corruption, and politician types then reflect different abilities to provide the public good or a distaste for corruption while in office.

We assume that the outcome distribution $F(\cdot \mid x)$ has a jointly differentiable density $f(y \mid x)$ and that for all $x \in X, F(\cdot \mid x)$ has full support on $Y=\mathbb{R}$. For simplicity we take the policy choice $x$ to be a shift parameter on the density of outcomes, so, abusing notation slightly, the density can be written $f(y \mid x)=f(y-x)$ for some fixed density $f(\cdot)$, and the probability that the realized outcome is less than $y$ given policy $x$ is simply $F(y-x)$. We assume that $f$ satisfies the standard monotone likelihood ratio property (MLRP), i.e.,

$$
\frac{f(y-x)}{f\left(y-x^{\prime}\right)}>\frac{f\left(y^{\prime}-x\right)}{f\left(y^{\prime}-x^{\prime}\right)}
$$

for all $x>x^{\prime}$ and all $y>y^{\prime}$. This implies that greater policy outcomes induce the voter to update favorably their beliefs about the policy adopted by the incumbent in the first period. As is well-known, the MLRP implies that the density function is unimodal, and that both the density and the distribution functions are strictly $\log$-concave. ${ }^{2}$ Moreover, we assume

$$
\lim _{y \rightarrow-\infty} \frac{f(y-x)}{f\left(y-x^{\prime}\right)}=\lim _{y \rightarrow+\infty} \frac{f\left(y-x^{\prime}\right)}{f(y-x)}=0 \text { when } x>x^{\prime},
$$

so that arbitrarily extreme signals become arbitrarily informative. As an example, $f(\cdot)$ may be a normal density.

\section{Electoral equilibrium}

As in the citizen-candidate model, we assume that neither the incumbent nor the challenger can make binding promises before an election. A related point is that we also assume that the voter cannot commit her vote, so that voting as well as policy making must be time consistent. Thus, our analysis focusses on perfect Bayesian equilibria of the electoral accountability model, under additional refinements to preclude implausible behavior on the part of the voter and politicians.

A strategy for the type $j$ incumbent is a pair $\pi_{j}=\left(\pi_{j}^{1}, \pi_{j}^{2}\right)$, where

$$
\pi_{j}^{1} \in \triangle(X) \quad \text { and } \quad \pi_{j}^{2}: X \times Y \rightarrow \triangle(X),
$$

\footnotetext{
${ }^{2}$ See Bagnoli and Bergstrom (2005) for an in-depth analysis of log concavity and related conditions.
} 
specifying mixtures over policy choices in period 1 and policy choices in period 2 for each possible previous policy choice and observed outcome. ${ }^{3}$ For tractability, we impose the restriction that the distribution $\pi_{j}^{1}$ has finite support for each type. A strategy for the type $j$ challenger is a mapping

$$
\gamma_{j}: Y \rightarrow \triangle(X),
$$

specifying mixtures over policy choices in period 2 for each policy type and observed outcome. A strategy for the voter is a mapping

$$
\rho: Y \rightarrow[0,1]
$$

where $\rho(y)$ is the probability of a vote for the incumbent given outcome $y$. A belief system for the voter is a probability distribution $\mu\left(\cdot \mid y_{1}\right)$ on $T \times X$ as a function of the observed outcome.

A strategy profile $\sigma=\left(\left(\pi_{j}, \gamma_{j}\right)_{j \in T}, \rho\right)$ is sequentially rational given belief system $\mu$ if neither the incumbent nor the challenger can gain by deviating from the proposed strategies at any decision node, and if the voter votes for the candidate that makes her best off in expectation following all possible realizations of $y_{1}{ }^{4}$ Beliefs $\mu$ are consistent with the strategy profile $\sigma$ if for every $y_{1}$, the distribution $\mu\left(j, x \mid y_{1}\right)$ is derived from $\left(\pi_{j}^{1}\right)_{j \in T}$ via Bayes' rule. A perfect Bayesian equilibrium is a pair $(\sigma, \mu)$ such that the strategy profile $\sigma$ is sequentially rational given the beliefs $\mu$, and $\mu$ is consistent with $\sigma$.

Sequential rationality implies that challengers will choose their ideal policies with probability one, since they cannot hope to be re-elected, so that $\gamma_{j}\left(\hat{x}_{j} \mid y_{1}\right)=1$ for all $y_{1}$. This implies that the expected payoff of electing the challenger for the voter is

$$
V^{C}=\sum_{k} p_{k} \mathbb{E}\left[u(y) \mid \hat{x}_{k}\right]
$$

Similarly, sequential rationality implies $\pi_{j}^{2}\left(\hat{x}_{j} \mid x_{1}, y_{1}\right)=1$ for all $x_{1}$ and all $y_{1}$, so the expected payoff to the voter from re-electing the incumbent is

$$
V^{I}\left(y_{1}\right)=\sum_{k} \mu_{T}\left(k \mid y_{1}\right) \mathbb{E}\left[u(y) \mid \hat{x}_{k}\right]
$$

where $\mu_{T}\left(j \mid y_{1}\right)$ is the marginal distribution of the incumbent's type given policy outcome $y_{1}$. Thus, the incumbent is re-elected if $V^{I}\left(y_{1}\right)>V^{C}$ and only if $V^{I}\left(y_{1}\right) \geqslant$

\footnotetext{
${ }^{3}$ Measurability of strategies or subsets of policies will be assumed implicitly, as needed, without further mention.

${ }^{4}$ In the terminology of Fearon (1999), voters focus on the problem of "selection," rather than "sanctioning." See his essay for arguments in support of this behavioral postulate.
} 
$V^{C}$. Sequential rationality does not pin down the ballot of the voter when she is indifferent between the incumbent and challenger; we say the equilibrium is deferential if the voter favors the incumbent when indifferent, so that the incumbent is re-elected if and only if $V^{I}\left(y_{1}\right) \geqslant V^{C}$.

This general formulation of deferential equilibrium implies that there is an $a c$ ceptance set of policy outcomes such that the incumbent is re-elected with probability one after realizations in this set and loses for sure after realizations outside the set:

$$
A=\left\{y_{1} \in Y: V^{I}\left(y_{1}\right) \geqslant V^{C}\right\}
$$

We say an equilibrium is monotonic if the acceptance set is closed, and if for every policy outcome belonging to the acceptance set, every greater outcome is also acceptable, i.e., for all $y \in A$ and all $y^{\prime} \geqslant y$, we have $y^{\prime} \in A$. Put differently, the voter follows a simple retrospective rule given by $\bar{y} \in \mathbb{R} \cup\{-\infty, \infty\}$ such that she re-elects the incumbent if and only if $y \geqslant \bar{y}$. The monotonicity condition imposes a natural linkage between the voter's utility over policy outcomes and the informational content of those outcomes in the first period.

Finally, an electoral equilibrium is a perfect Bayesian equilibrium that is deferential and monotonic. Electoral equilibria are then characterized by three conditions. First, the threshold $\bar{y}$ must be such that, anticipating that politicians choose their ideal policies in the second period, the expected utility of re-electing the incumbent conditional on observing $y$ is greater than or equal to $\sum_{k} p_{k} \mathbb{E}\left[u(y) \mid \hat{x}_{k}\right]$ if and only if $y \geqslant \bar{y}$. Second, each politician type $j$, knowing that she is re-elected if and only if $y \geqslant \bar{y}$, mixes over optimal actions in the first period, i.e., the type $j$ incumbent's policy strategy $\pi_{j}$ places probability one on maximizers of

$$
w_{j}(x)+(1-F(\bar{y}-x))\left[w_{j}\left(\hat{x}_{j}\right)+\beta\right]+F(\bar{y}-x) V^{C} .
$$

Third, updating of voter beliefs follows Bayes rule, i.e., after observing outcome $y$, the voter's posterior beliefs assign probability

$$
\mu_{T}(j \mid y)=\frac{p_{j} \sum_{x} f(y-x) \pi_{j}(x)}{\sum_{k} p_{k} \sum_{x} f(y-x) \pi_{k}(x)}
$$

to the incumbent being type $j$. Since the outcome density is positive, every outcome is on the path of play, so Bayes' rule pins down the voter's beliefs. We henceforth summarize an electoral equilibrium by the strategy profile $\sigma$, leaving beliefs implicit. 


\section{Preliminary analysis}

To facilitate the analysis, we assume that all incumbent types are in principle interested in re-election, i.e.,

$$
w_{1}\left(\hat{x}_{1}\right)+\beta>V^{C}
$$

so that if re-election is assured by choosing their ideal policies in the first period, then the benefits of re-election outweigh the costs. Note that the incumbent can always choose her ideal policy, so it is never optimal for the politician to choose large policies $x$ for which $w_{j}(x)+\beta<\mathbb{E}\left[u(y) \mid \hat{x}_{j}\right]$. By (C3) and monotonicity, it is never optimal to choose a policy below the politician's ideal policy, so there is at least one solution to the incumbent's problem in the first period. Denoting by $x_{j}^{*}$ such a solution, the necessary first order condition for a solution of the incumbent's maximization problem (1) is

$$
w_{j}^{\prime}\left(x_{j}^{*}\right)=-f\left(\bar{y}-x_{j}^{*}\right)\left[w_{j}\left(\hat{x}_{j}\right)+\beta-V^{C}\right] .
$$

That is, the marginal disutility in the current period from increasing the policy choice is just offset by the marginal utility in the second period, owing to the politician's increased chance of re-election. By (C3), the right-hand side of (2) is negative, and we see that for an arbitrary cutoff $\bar{y}$, the politician optimally exerts a positive amount of effort, i.e., chooses $x_{j}^{*}>\hat{x}_{j}$, in the first term of office.

We can gain some insight into the incumbent's problem by reformulating it in terms of optimization subject to an inequality constraint. Define a new objective function

$$
U_{j}(x, r)=w_{j}(x)+r\left[w_{j}\left(\hat{x}_{j}\right)+\beta-V^{C}\right],
$$

which is the expected utility if the politician chooses policy $x$ and is re-elected with probability $r$, minus a constant term corresponding to the current enjoyment of office. Note that $U_{j}$ is concave in $(x, r)$ and quasi-linear in $r$. Of course, given $x$, the re-election probability is in fact pinned down as $1-F(\bar{y}-x)$. Defining the constraint function

$$
g(x, r)=1-F(\bar{y}-x)-r,
$$

we can then formulate the politician's optimization problem as

$$
\begin{gathered}
\max _{(x, r)} U_{j}(x, r) \\
\text { s.t. } g(x, r) \leqslant 0,
\end{gathered}
$$




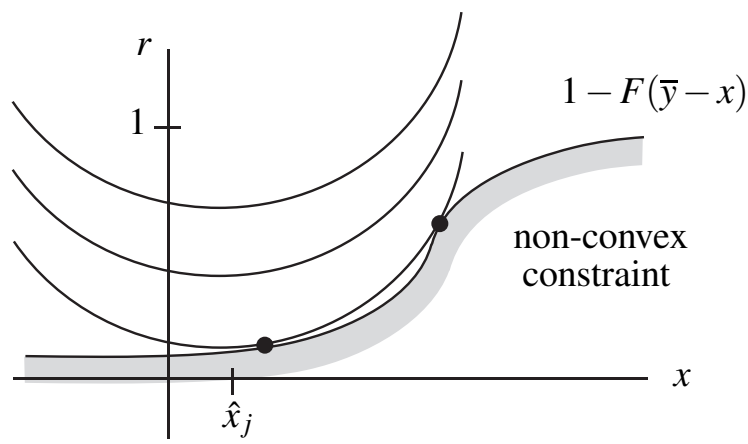

Figure 2: Politician's optimization problem

which has the general form depicted in Figure 2. Here, the objective function is well-behaved, but the constraint set inherits the natural non-convexity of the distribution function $F$, leading to the possibility of multiple solutions. This, in turn, can lead to multiple optimal policies and the necessity of mixing in equilibrium; see Figure 2 for an illustration of this multiplicity.

We exploit log concavity and impose further restrictions on the risk aversion of politicians to limit the need for mixing to at most two policy choices for each type. ${ }^{5}$ Assume that for all $j$, all finite $\bar{y}$, and all $x, \tilde{x}, z$ with $\hat{x}_{j}<x<\tilde{x}<z$, we have

$$
\text { if } \begin{aligned}
\frac{w_{j}^{\prime \prime}(x)}{w_{j}^{\prime}(x)} \leqslant- & \frac{f^{\prime}(\bar{y}-x)}{f(\bar{y}-x)} \text { and } \frac{w_{j}^{\prime \prime}(z)}{w_{j}^{\prime}(z)} \leqslant-\frac{f^{\prime}(\bar{y}-z)}{f(\bar{y}-z)}, \\
& \text { then } \frac{w_{j}^{\prime \prime}(\tilde{x})}{w_{j}^{\prime}(\tilde{x})}<-\frac{f^{\prime}(\bar{y}-\tilde{x})}{f(\bar{y}-\tilde{x})}
\end{aligned}
$$

That is, the set of $x>\hat{x}_{j}$ such that $w_{j}^{\prime \prime}(x) / w_{j}^{\prime}(x) \leqslant-f^{\prime}(\bar{y}-x) / f(\bar{y}-x)$ is convex, and if $x$ and $z$ satisfy the inequality, then every policy between them satisfies it strictly. To understand this condition, note that by $\log$ concavity of $f(\cdot)$, the term $f^{\prime}(\bar{y}-x) / f(\bar{y}-x)$ is strictly decreasing in $x$, and thus (C4) is satisfied if the coefficient of absolute risk aversion, $w_{j}^{\prime \prime}(x) / w_{j}^{\prime}(x)$, does not decrease too fast to the right of the type $j$ politicians' ideal policy. To illustrate, when the utility function $w_{j}$ is quadratic, the coefficient of absolute risk aversion is $\left(x-\hat{x}_{j}\right)^{-1}$, and when the density $f$ is standard normal, the likelihood ratio $f^{\prime}(\bar{y}-x) / f(\bar{y}-x)$ simplifies to $\bar{y}-x$. Thus, (C4) is satisfied in the quadratic-normal special case, depicted in Fig-

\footnotetext{
${ }^{5}$ The possibility of multiple optimizers has a counterpart in static models of elections with probabilistic voting, where log concavity is sufficient to ensure existence of equilibria in pure strategies (cf. Roemer (1997) and Bernhardt et al. (2009)).
} 


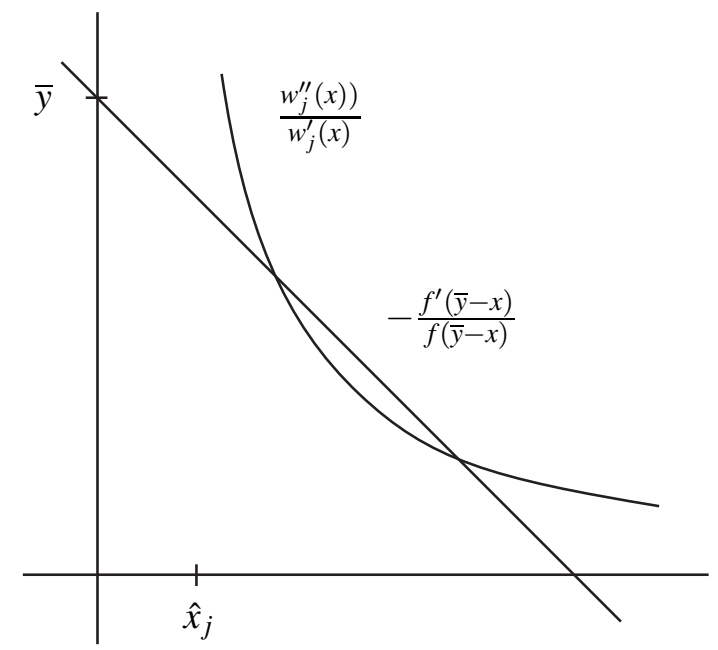

Figure 3: Quadratic-normal special case

ure 3. Likewise, in the case of exponential utility, the coefficient of risk aversion is $\left(1-\exp \left(\hat{x}_{j}-x\right)\right)^{-1}$, and again $(\mathrm{C} 4)$ is satisfied.

The usefulness of (C4) is delineated in the next result, which implies that for arbitrary cutoffs, each type of incumbent has at most two optimal policies as a function of the cutoff. The greater solution to the incumbent's optimization problem, which is denoted $x_{j}^{*}(\bar{y})$, corresponds to "going for broke," while the least solution, denoted $x_{*, j}(\bar{y})$, corresponds to "taking it easy." When these two policy choices coincide, the politician has a unique optimal policy; a gap between the two choices reflects the possibility that the increase in effort involved in going for broke is just offset by the increase in probability of being re-elected. Of course, standard continuity arguments imply that the correspondence of optimal policies has closed graph; in the present context, this means that the functions $x_{j}^{*}(\cdot)$ and $x_{*, j}(\cdot)$ are, respectively, upper and lower semi-continuous.

Proposition 1. Assume $(\mathrm{CO})-(\mathrm{C} 4)$. For every cutoff $\bar{y} \in Y$ and every type $j$, there are at most two local maximizers of the objective function (1), and the greatest and least optimal policies, $x_{j}^{*}(\bar{y})$ and $x_{*, j}(\bar{y})$, are upper semi-continuous and lower semi-continuous, respectively, as a function of the cutoff.

Proof. Suppose there are three distinct local maximizers of the type $j$ politicians' objective function, say $x^{\prime}, x^{\prime \prime}$, and $x^{\prime \prime \prime}$ with $x^{\prime}<x^{\prime \prime}<x^{\prime \prime \prime}$. Thus, there are local minimizers $z^{\prime}$ and $z^{\prime \prime}$ such that $x^{\prime}<z^{\prime}<x^{\prime \prime}<z^{\prime \prime}<x^{\prime \prime \prime}$. With (C3), inspection of the first order condition (2) at $x$ reveals that $w_{j}^{\prime}\left(z^{\prime}\right)<0$ and $w_{j}^{\prime}\left(z^{\prime \prime}\right)<0$, and we can 
rewrite the first order condition at $z^{\prime}$ and $z^{\prime \prime}$ as

$$
w_{j}\left(\hat{x}_{j}\right)+\beta-V^{C}=-\frac{w_{j}^{\prime}\left(z^{\prime}\right)}{f\left(\bar{y}-z^{\prime}\right)}=-\frac{w_{j}^{\prime}\left(z^{\prime \prime}\right)}{f\left(\bar{y}-z^{\prime \prime}\right)} .
$$

By the necessary second order condition for a local minimizer, the second derivative at $z^{\prime}$ satisfies

$0 \leqslant w_{j}^{\prime \prime}\left(z^{\prime}\right)-f^{\prime}\left(\bar{y}-z^{\prime}\right)\left[w_{j}\left(\hat{x}_{j}\right)+\beta-V^{C}\right]=w_{j}^{\prime \prime}\left(z^{\prime}\right)-f^{\prime}\left(\bar{y}-z^{\prime}\right)\left[-\frac{w_{j}^{\prime}\left(z^{\prime}\right)}{f\left(\bar{y}-z^{\prime}\right)}\right]$,

or equivalently,

$$
\frac{w_{j}^{\prime \prime}\left(z^{\prime}\right)}{w_{j}^{\prime}\left(z^{\prime}\right)} \leqslant-\frac{f^{\prime}\left(\bar{y}-z^{\prime}\right)}{f\left(\bar{y}-z^{\prime}\right)}
$$

Similarly, we have

$$
\frac{w_{j}^{\prime \prime}\left(z^{\prime \prime}\right)}{w_{j}^{\prime}\left(z^{\prime \prime}\right)} \leqslant-\frac{f^{\prime}\left(\bar{y}-z^{\prime \prime}\right)}{f\left(\bar{y}-z^{\prime \prime}\right)} .
$$

Since $x^{\prime \prime}$ is a local maximizer, the first order condition holds at $x^{\prime \prime}$, and the second derivative at $x^{\prime \prime}$ is non-positive, but then we have

$$
\frac{w_{j}^{\prime \prime}\left(x^{\prime \prime}\right)}{w_{j}^{\prime}\left(x^{\prime \prime}\right)} \geqslant-\frac{f^{\prime}\left(y-x^{\prime \prime}\right)}{f\left(y-x^{\prime \prime}\right)}
$$

contradicting (C4). We conclude that the objective function has at most two local maximizers.

We can illustrate Proposition 1 assuming the normal density and exponential utility, say $w_{j}(x)=-e^{x}+1$. Then the first order condition is

$$
-e^{x}=-\frac{\Delta}{\sigma \sqrt{2 \pi}} e^{-\frac{(\bar{y}-x)^{2}}{2 \sigma^{2}}}
$$

where $\Delta=\beta-w_{j}\left(\hat{x}_{j}\right)-V^{C}=\beta-V^{C}>0$. Multiplying by negative one and taking $\log$ s of both sides, this is a quadratic equation in $x$, with solutions

$$
x=\bar{y}+\mu-\sigma^{2} \pm \sigma \sqrt{\sigma^{2}-2(\bar{y}+\mu)+2 \ln \left(\frac{\Delta}{\sigma \sqrt{2 \pi}}\right)} .
$$

The solutions are real as long as office benefit is sufficiently high relative to the cutoff, and otherwise there is no solution to the first order condition, so that the 
politician optimizes at the corner by choosing zero effort. Alternatively, the solutions are real if the variance of the observed outcome is sufficiently small. Note that the optimal effort increases without bound as the variance becomes small or the office benefit becomes large; we return to this theme in our analysis of responsive democracy, in Section 6.

The next proposition establishes that the incumbent's objective function satisfies the important property that differences in payoffs are monotone in type. We say that $U_{j}(x, 1-F(\bar{y}-x))$ is supermodular in $(j, x)$ if for all $(j, x)$ and all $(k, z)$ with $j>k$ and $x>z$, we have

$$
\begin{aligned}
& U_{j}(x, 1-F(\bar{y}-x))-U_{j}(z, 1-F(\bar{y}-z)) \\
& \quad>U_{k}(x, 1-F(\bar{y}-x))-U_{k}(z, 1-F(\bar{y}-z)) .
\end{aligned}
$$

An implication is that given an arbitrary value $\bar{y}$ of the cutoff, the optimal policy choices of the types are strictly ordered by type, i.e.,

$$
\text { for all } j<n, \quad x_{j}^{*}(\bar{y})<x_{*, j+1}(\bar{y}) .
$$

This ordering property will, in turn, be critical for establishing existence of equilibrium.

Proposition 2. Assume (CO)-(C4). For every cutoff $\bar{y}$, the incumbent's objective function, $U_{j}(x, 1-F(\bar{y}-x))$, is super modular in $(j, x)$.

Proof. Consider $j>k$ and $x>z$, and rewrite the inequality in the definition of supermodularity as

$$
\begin{aligned}
& \theta_{j}(v(x)-v(z))+(F(\bar{y}-z)-F(\bar{y}-x))\left(\theta_{j} v\left(\hat{x}_{j}\right)-c\left(\hat{x}_{j}\right)\right) \\
& \quad>\theta_{k}(v(x)-v(z))+(F(\bar{y}-z)-F(\bar{y}-x))\left(\theta_{k} v\left(\hat{x}_{k}\right)-c\left(\hat{x}_{k}\right)\right) .
\end{aligned}
$$

Since $\theta_{j}>\theta_{k}$ and $v(x)>v(z)$ it suffices to show

$$
(F(\bar{y}-z)-F(\bar{y}-x))\left(\theta_{j} v\left(\hat{x}_{j}\right)-c\left(\hat{x}_{j}\right)\right)>(F(\bar{y}-z)-F(\bar{y}-x))\left(\theta_{k} v\left(\hat{x}_{k}\right)-c\left(\hat{x}_{k}\right)\right) .
$$

Since $F(\bar{y}-z)>F(\bar{y}-x)$ and $\theta_{j} v\left(\hat{x}_{j}\right)-c\left(\hat{x}_{j}\right)>\theta_{k} v\left(\hat{x}_{k}\right)-c\left(\hat{x}_{k}\right)$, the desired inequality indeed holds.

The above ordering property is very useful in combination with the fact that given arbitrary policy choices $x_{1}<x_{2}<\cdots<x_{n}$ of the politician types in the first period, there is a unique outcome, which we denote $y^{*}\left(x_{1}, \ldots, x_{n}\right)$, such that conditional on realizing this value, the voter is indifferent between re-electing the incumbent and electing a challenger. Moreover, this extends to the case of mixed 
policy strategies $\pi_{1}, \ldots, \pi_{n}$ with supports that are strictly ordered by type, i.e., for all $j<n$,

$$
\max \left\{x: \pi_{j}(x)>0\right\}<\min \left\{x: \pi_{j+1}(x)>0\right\} .
$$

That is, there is a unique solution in $\bar{y}$ to the equation $V^{I}(\bar{y})=V^{C}$, or more explicitly,

$$
\sum_{k} \mu_{T}(k \mid \bar{y}) \mathbb{E}\left[u(y) \mid \hat{x}_{k}\right]=\sum_{k} p_{k} \mathbb{E}\left[u(y) \mid \hat{x}_{k}\right]
$$

We let $y^{*}\left(\pi_{1}, \ldots, \pi_{n}\right)$ denote the solution to the voter's indifference condition as a function of policy choices.

In addition to uniqueness, the next proposition establishes that the cutoff is continuous in policy strategies and lies between the choices of the type 1 and type $n$ politicians, shifted by the mode of the density of $f(\cdot)$, which we denote by $\hat{z}$.

Proposition 3. Assume (CO)-(C2). For all mixed policy strategies $\pi_{1}, \ldots, \pi_{n}$ with supports that are strictly ordered by type and for all belief systems $\mu$ derived via Bayes rule, there is a unique solution to the voter's indifference condition (3), and the solution $y^{*}\left(\pi_{1}, \ldots, \pi_{n}\right)$ is continuous as a function of mixed policies. Moreover, this solution lies between the extreme policy choices shifted by the mode of the outcome density, i.e.,

$$
\min \left\{x: \pi_{1}(x)>0\right\}+\hat{z} \leqslant y^{*}\left(\pi_{1}, \ldots, \pi_{n}\right) \leqslant \max \left\{x: \pi_{n}(x)>0\right\}+\hat{z} .
$$

Proof. For existence of a solution to the indifference condition, fix $\pi_{1}, \ldots, \pi_{n}$ with supports that are strictly ordered by type, and note that the left-hand side of (3) is continuous in $\bar{y}$. For any $j<n$, let $x_{j}=\max \left\{x: \pi_{j}(x)>0\right\}$ be the greatest policy chosen with positive probability by the type $j$ politicians, and let $x_{n}=\min \{x$ : $\left.\pi_{n}(x)>0\right\}$ be the lowest policy chosen with positive probability by the type $n$ politicians. For all $j$ and all $x<x_{j}$ with $\pi_{j}(x)>0$, (C1) implies that for sufficiently large $\bar{y}$, we have $f(\bar{y}-x)<f\left(\bar{y}-x_{j}\right)$. Then (C1) and (C2) imply

$$
\mu_{T}(j \mid \bar{y})=\frac{p_{j} \sum_{x} f(\bar{y}-x) \pi_{j}(x)}{\sum_{k} p_{k} \sum_{x} f(\bar{y}-x) \pi_{k}(x)} \leqslant \frac{p_{j}}{\sum_{k} p_{k} \sum_{x} \frac{f(\bar{y}-x)}{f\left(\bar{y}-x_{j}\right)} \pi_{k}(x)} \leqslant \frac{p_{j}}{p_{n} \frac{f\left(\bar{y}-x_{n}\right)}{f\left(\bar{y}-x_{j}\right)}} \rightarrow 0
$$

as $\bar{y} \rightarrow \infty$, which implies that $\mu_{T}(n \mid \bar{y})$ goes to one as the cutoff increases. In words, when the policies of the politicians are ordered by type, high realizations of the outcome become arbitrarily strong evidence that the incumbent is the best possible type. Similarly, $\mu_{T}(1 \mid \bar{y})$ goes to one as $\bar{y}$ decreases without bound. Thus, the lefthand side of (3) approaches $\mathbb{E}\left[u(y) \mid \hat{x}_{n}\right]$ when the cutoff is large, and it approaches 
$\mathbb{E}\left[u(y) \mid \hat{x}_{1}\right]$ when the cutoff is small, and existence of a solution follows from the intermediate value theorem. Uniqueness follows from the fact that the left-hand side is strictly increasing in $\bar{y}$, from Lemma A.6 of Banks and Sundaram (1998). Standard continuity arguments imply that $y^{*}\left(\pi_{1}, \ldots, \pi_{n}\right)$ is continuous in its arguments.

To obtain the upper bound on the cutoff, consider any $\bar{y}>\max \left\{x: \pi_{n}(x)>\right.$ $0\}+\hat{z}$. Recall that the posterior probability that the politician is type $j$, conditional on observing $\bar{y}$, is

$$
\mu_{T}(j \mid \bar{y})=\frac{p_{j} \sum_{x} f(\bar{y}-x) \pi_{j}(x)}{\sum_{k} p_{k} \sum_{x} f(\bar{y}-x) \pi_{k}(x)} .
$$

Note that for all $k>j$ and all policies $x_{j}$ with $\pi_{j}\left(x_{j}\right)>0$ and $x_{k}$ with $\pi_{k}\left(x_{k}\right)>0$, we have $\hat{z}<\bar{y}-x_{k}<\bar{y}-x_{j}$. Since $f(\cdot)$ is single-peaked by (C1), we see that for all $x_{1}, \ldots, x_{n}$ such that each $x_{k}$ is in the support of $\pi_{k}$, we have

$$
f\left(\bar{y}-x_{1}\right)<f\left(\bar{y}-x_{2}\right)<\cdots<f\left(\bar{y}-x_{n}\right) .
$$

Therefore, the coefficients on prior beliefs are ordered by type, i.e.,

$$
\frac{\sum_{x} f(\bar{y}-x) \pi_{1}(x)}{\sum_{k} p_{k} \sum_{x} f(\bar{y}-x) \pi_{k}(x)}<\cdots<\frac{\sum_{x} f(\bar{y}-x) \pi_{n}(x)}{\sum_{k} p_{k} \sum_{x} f\left(\bar{y}-x_{k}\right) \pi_{k}(x)},
$$

and we conclude that the posterior distribution $\mu_{T}(\cdot \mid \bar{y})$ first order stochastically dominates the prior, contradicting the indifference condition. An analogous argument derives a contradiction for the case $\bar{y}<\min \left\{x: \pi_{1}(x)>0\right\}+\hat{z}$.

To see the structure of $y^{*}\left(\pi_{1}, \ldots, \pi_{n}\right)$ for the special case of two types using pure policy strategies, the voter's cutoff is simply the solution to $\mu_{T}(2 \mid y)=p_{2}$, so that conditional on the cutoff, the probability that the incumbent is the high type is just equal to the prior probability. Letting $x_{1}$ and $x_{2}$ be the policies chosen by the two types, this means that $y^{*}\left(x_{1}, x_{2}\right)$ solves the equation

$$
p_{2}=\frac{p_{2} f\left(y-x_{2}\right)}{p_{1} f\left(y-x_{1}\right)+p_{2} f\left(y-x_{2}\right)},
$$

or after manipulating, it means that the likelihood of $y$ is the same given the policy choices of the politician types, i.e., $f\left(y-x_{1}\right)=f\left(y-x_{2}\right)$. Adding the assumption that the density $f(\cdot)$ is standard normal, the cutoff is simply the midpoint of the politicians' choices, i.e.,

$$
y^{*}\left(x_{1}, x_{2}\right)=\frac{x_{1}+x_{2}}{2} .
$$




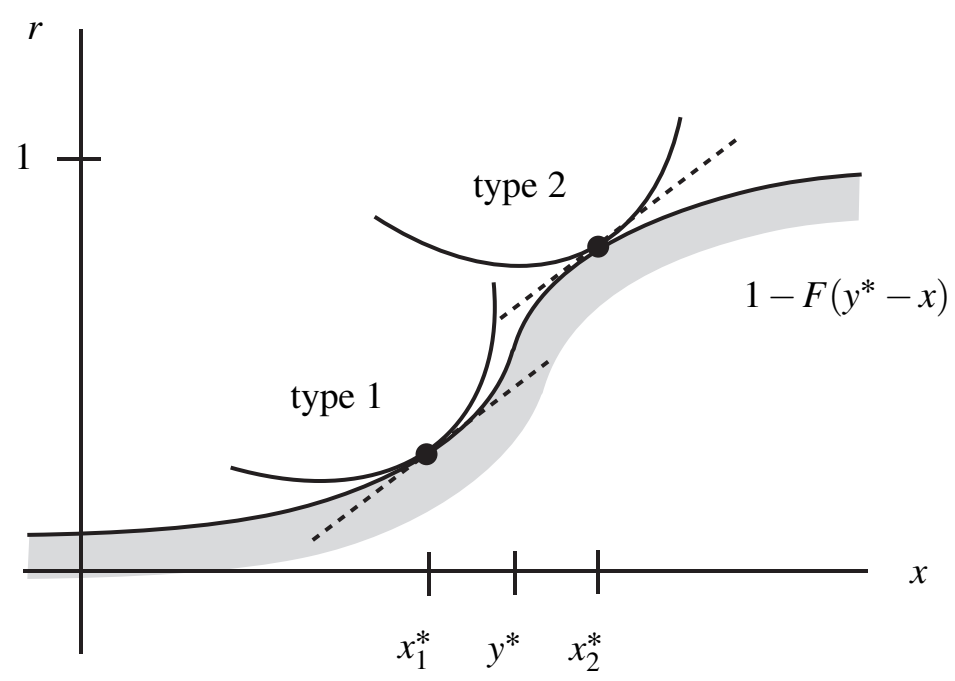

Figure 4: Electoral equilibrium

Indeed, this characterization as the midpoint of policy choices extends to any density that is symmetric around zero.

The preceding observations allow us to graphically depict an electoral equilibrium in pure policy strategies for the case of two types. In Figure 4, we draw the indifference curves of $U_{1}$ and $U_{2}$ through the unique optimal policies, $x_{1}^{*}$ and $x_{2}^{*}$, of the politician types given the constraint set determined by the cutoff $y^{*}$. This is reflected in the tangency condition at each optimal policy. Moreover, the voter's indifference condition implies that the likelihood of outcome $y^{*}$ is equal given either optimal policy, and this implies that the two tangent lines have equal slopes. Indeed, using the first order condition for office holders of types 1 and 2, we have

$$
\frac{w_{1}^{\prime}\left(x_{1}^{*}\right)}{w_{1}\left(\hat{x}_{1}\right)+\beta-V^{C}}=-f\left(y^{*}-x_{1}^{*}\right)=-f\left(y^{*}-x_{2}^{*}\right)=\frac{w_{2}^{\prime}\left(x_{2}^{*}\right)}{w_{2}\left(\hat{x}_{2}\right)+\beta-V^{C}},
$$

as claimed. Note that when the office benefit $\beta$ increases, the indifference curves of the politician types become flatter, and optimal policies will move to the right, suggesting that higher office benefit leads to greater policy responsiveness.

\section{Existence and characterization of electoral equilibria}

Our first main result establishes existence of electoral equilibrium, along with a partial characterization of equilibria. Importantly, even in this two-period model 
of elections (where second-period policies are pinned down by end-game effects), electoral equilibria must solve a complicated fixed point problem: optimal policy choices of politician types depend on the cutoff used by the voter, and the cutoff used by the voter depends, via Bayes rule, on the policy choices of politician types. Nevertheless, we rely on Propositions 1-3 to provide a fixed point argument and overcome the existence problem.

Theorem 5.1. Assume $(\mathrm{CO})-(\mathrm{C} 4)$. Then there is an electoral equilibrium, and every electoral equilibrium is given by mixed policy strategies $\pi_{1}^{*}, \ldots, \pi_{n}^{*}$ and a finite cutoff $y^{*}$ such that:

(i) each type $j$ politician mixes over policies using $\pi_{j}^{*}$, which places positive probability on at most two policies, say $x_{j}^{*}$ and $x_{*, j}$, where $\hat{x}_{j}<x_{*, j} \leqslant x_{j}^{*}$,

(ii) the supports of policy strategies are strictly ordered by type, i.e., for all $j<n$, we have $x_{j}^{*}<x_{*, j+1}$,

(iii) the voter re-elects the incumbent if and only if $y \geqslant y^{*}$, where the cutoff lies between the extreme policies shifted by the mode of the outcome density, i.e., $x_{*, 1}+\hat{z} \leqslant y^{*} \leqslant x_{n}^{*}+\hat{z}$.

Proof. In proving the proposition, we must address two technical subtleties. The first is that when supports of mixed policy choices are only weakly ordered, the left-hand side of (3) is only weakly increasing, so that the equality has a closed, convex (not necessarily singleton) set of solutions. In fact, if all politician types choose the same policy with probability one, then updating does not occur and incumbents are always re-elected, so that the voter's cutoff is negatively infinite. As policy choices of politician types converge to the same policy, this means that the cutoff either jumps discontinuously (from a bounded, finite level) or diverges to negative infinity. We circumvent this problem by deriving a positive lower bound on the distance between optimal policy choices of the different types. Indeed, we first observe that equilibrium policy choices are bounded above by any choice $\bar{x}$ such that $\mathbb{E}\left[u(y) \mid \hat{x}_{n}\right]>w_{n}(\bar{x})+\beta$, i.e., $-w_{n}(\bar{x})>\beta-\mathbb{E}\left[u(y) \mid \hat{x}_{n}\right]$. That is, if the type $n$ politician prefers to choose her ideal policy with no chance of re-election rather than choose $\bar{x}$ and win with certainty, then no policy above $\bar{x}$ can be optimal for any type given any cutoff.

Next, given any cutoff $\bar{y}$ and any type $j$ politician, there are at most two optimal policies, by Proposition 1, and each satisfies the first order condition (2). Note that $f(\bar{y}-x) \rightarrow 0$ uniformly on $[0, \bar{x}]$ as $|\bar{y}| \rightarrow \infty$, and from the first order condition, this implies that the optimal policies of the type $j$ politician converge to the ideal policy, i.e., $x_{j}^{*}(\bar{y}) \rightarrow \hat{x}_{j}$ and $x_{*, j}(\bar{y}) \rightarrow \hat{x}_{j}$. Thus, we can choose a sufficiently large interval 
$\left[y_{L}, y_{H}\right]$ and $\varepsilon^{\prime}>0$ such that for all $\bar{y}$ outside the interval, optimal policies differ across types by at least $\varepsilon^{\prime}$, i.e., for all $j<n$, we have $\left|x_{*, j+1}(\bar{y})-x_{j}^{*}(\bar{y})\right|>\varepsilon^{\prime}$. By upper semi-continuity of $x_{j}^{*}(\cdot)$ and lower semi-continuity of $x_{*, j+1}(\cdot)$, the function $\left|x_{*, j+1}(\bar{y})-x_{j}^{*}(\bar{y})\right|$ attains its minimum on $\left[y_{L}, y_{H}\right]$, and this minimum is positive. Thus, there exists $\varepsilon^{\prime \prime}>0$ such that for all $\bar{y} \in\left[y_{L}, y_{H}\right]$, optimal policies differ by at least $\varepsilon^{\prime \prime}$. Finally, we set $\varepsilon=\min \left\{\varepsilon^{\prime}, \varepsilon^{\prime \prime}\right\}$ to establish the desired lower bound.

We are interested in the profiles $\left(\pi_{1}, \ldots, \pi_{n}\right)$ such that for all politician types $j, \pi_{j}$ places positive probability on at most two alternatives, and the supports of mixed policy strategies are strictly ordered by type and separated by a distance of at least $\varepsilon$, i.e., for all $j<n$ and all policies $x_{j}$ with $\pi_{j}\left(x_{j}\right)>0$ and $x_{j+1}$ with $\pi_{j+1}\left(x_{j+1}\right)>0$, we have $x_{j}+\varepsilon \leqslant x_{j+1}$. It is convenient to represent such a profile by a $3 n$-tuple $(x, z, r)$, where $x=\left(x_{1}, \ldots, x_{n}\right) \in[0, \bar{x}]^{n}, z=\left(z_{1}, \ldots, z_{n}\right) \in[0, \bar{x}]^{n}$, and $r=\left(r_{1}, \ldots, r_{n}\right) \in[0,1]^{n}$. In addition, we require that for all $j$, we have $x_{j} \leqslant z_{j}$, and that for all $j<n$, we have $z_{j}+\varepsilon \leqslant x_{j+1}$. We then associate $(x, z, r)$ with the profile of mixed policy strategies such that the type $j$ politician places probability $r_{j}$ on $x_{j}$ and the remaining probability $1-r_{j}$ on $z_{j}$. Letting $D^{\varepsilon}$ consist of all such $3 n$-tuples $(x, z, r)$, we see that $D^{\varepsilon}$ is nonempty, convex, and compact. Using this representation, we can define (abusing notation slightly) the induced cutoff $y^{*}(x, z, r)$, which is continuous as a function of its arguments.

The second difficulty is that the set $Y$ of policy outcomes is not compact, so that the voter's cutoff is, in principle, unbounded. To circumvent this problem, we note that by continuity of the function $y^{*}(\cdot)$ the image $y^{*}\left(D^{\varepsilon}\right)$ is compact, and we can let $\bar{Y}$ be a convex, compact set containing this image. The existence proof then proceeds with an application of Kakutani's fixed point theorem. We define the correspondence $\Phi: D^{\varepsilon} \times \bar{Y} \rightrightarrows D^{\varepsilon} \times \bar{Y}$ so that for each $(x, z, r, \bar{y})$, the value of $\Phi$ consists of $(3 n+1)$-tuples $(\tilde{x}, \tilde{z}, \tilde{r}, \tilde{y})$ such that for every politician type $j$, the policies $\tilde{x}_{j}$ and $\tilde{z}_{j}$ are optimal and $\tilde{y}$ is the unique cutoff induced by the indifference condition:

$\Phi(x, z, r, \bar{y})=\left\{(\tilde{x}, \tilde{z}, \tilde{r}, \tilde{y}) \in D^{\varepsilon} \times \bar{Y} \mid \begin{array}{c}\text { for all } j, \tilde{x}_{j}=x_{*, j}(\bar{y}) \text { and } \tilde{z}_{j}=x_{j}^{*}(\bar{y}) \\ \text { and } \tilde{y}=y^{*}(x, z, r)\end{array}\right\}$.

This correspondence is upper hemi-continuous with convex, closed values, and the domain $D^{\varepsilon} \times \bar{Y}$ is nonempty, compact, and convex. Therefore, Kakutani's theorem implies that $\Phi$ has a fixed point, $\left(x^{*}, z^{*}, r^{*}, y^{*}\right)$, which yields an electoral equilibrium. Finally, the characterization results in (i)-(iii) follow directly from Propositions 1-3.

We have assumed that the policy space $X=\mathbb{R}_{+}$is unbounded above, and this has facilitated the development by removing the possibility of corner solutions and permitting a first order analysis. Of course, an equilibrium with highest policy 


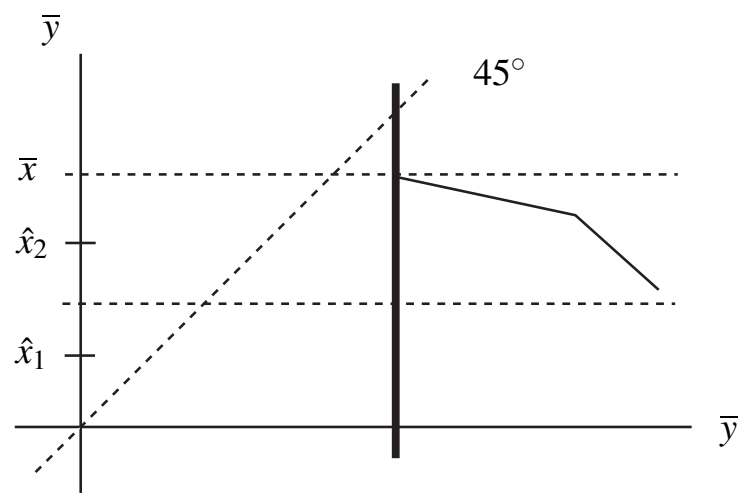

Figure 5: Existence problems with bounded policy

choice $x_{n}^{*}$ survives if we modify the model by imposing an upper bound $\bar{x} \geqslant x_{n}^{*}$ on feasible policies. But imposing such a bound a priori, and independent of the office benefit, creates serious technical difficulties stemming from the possibility that all types pool at the upper bound, which implies that the voter does not revise her prior beliefs after observing the outcome $y$. Specifically, this difficulty arises when the office benefit is large and incumbent types have strong incentives to exert higher effort to improve their chances of re-election. The problem is illustrated in Figure 5, where we suppose there are just two types. When the voter's cutoff $\bar{y}$ is large, the optimal policies of the politicians will be close to their ideal policies. Then, if the density $f$ is symmetric around zero, the induced cutoff defined by the voter's indifference condition will be roughly the midpoint between the optimal policies. As we decrease the cutoff, the optimal policies increase, and so does the induced cutoff; this relationship is represented by the kinked line in the figure. At some point, the type 2 politicians' optimal policy hits the upper bound $\bar{x}$, and it is possible that the type 1 also hits the upper bound before the induced cutoff crosses the $45^{\circ}$ line. At that point, both types are at a corner solution, and the voter does not update-so the induced cutoff jumps to negative infinity. That is, a deferential voting strategy would imply that the voter re-elect the incumbent no matter what, which of course is incompatible with equilibrium.

\section{Responsive democracy}

We have not yet touched on the possibility of responsive democracy, meaning that incumbents choose high levels of policy, despite short run incentives to choose their ideal policy. Given the short time horizon (and limited ability of the voter 
to sanction politicians), and given the divergence in preferences between the voter and politicians, the prospects for well-functioning democratic elections may seem dim. Nevertheless, when $\beta$ is large, so that politicians are substantially officemotivated, we obtain a form of the responsive democracy result. We now make use of a standard Inada-type condition that is satisfied in the quadratic and exponential cases and many other cases of interest: for all $j$,

$$
\lim _{x \rightarrow \infty} w_{j}^{\prime}(x)=-\infty
$$

Let $G=\left\{j: \mathbb{E}\left[u(y) \mid \hat{x}_{j}\right]>V^{C}\right\}$ denote the set of above average types, which are such that the expected utility from their ideal policy exceeds the expected utility from a challenger. Let $\ell=\min G$ be the smallest above average type.

Our second, and final, main result provides a characterization of equilibria when office benefit is high. We find that the voter becomes arbitrarily demanding, in the sense that the equilibrium cutoff diverges to infinity, that the policy choices of all politician types become close to their ideal policy or arbitrarily large, and that all above average types in fact exert unbounded effort. An immediate implication, since type $n$ is above average and $p_{n}>0$, is that the voter's expected utility from politicians' choices in the first period increases without bound as office benefit becomes large, i.e.,

$$
\sum_{j} p_{j} \sum_{x} \mathbb{E}[u(y) \mid x] \pi_{j}(x) \rightarrow \infty .
$$

It is possible that some politician type mixes between a policy that is close to the ideal policy and another that becomes arbitrarily large, but because policy choices are ordered by type, an implication of the proposition is that this can obtain for at most one politician type; choices of lower types will converge to their ideal policies, while choices of higher types will diverge to infinity. Note that the Inada condition (C5) is used only to prove part (iii) of the result.

Theorem 6.1. Assume (CO)-(C5). Let the office benefit $\beta$ be arbitrarily large. Then for every selection of electoral equilibria $\sigma$, the voter's cutoff diverges to infinity; for each politician type $j$, the policy choices of all above average types increase without bound; and the greatest policy choice of other types either increases without bound or accumulates at the ideal policy:

(i) $y^{*} \rightarrow \infty$,

(ii) for all $j$, all $\varepsilon>0$, and sufficiently large $\beta$, we have $\left\{x_{*, j}, x_{j}^{*}\right\} \subseteq\left(\hat{x}_{j}, \hat{x}_{j}+\varepsilon\right) \cup$ $\left(\frac{1}{\varepsilon}, \infty\right)$ 
(iii) $x_{\ell-1}^{*} \rightarrow \infty$, and thus for all $j \geqslant \ell$, we have $x_{*, j} \rightarrow \infty$.

Proof. Let $\beta$ be large, and let $\sigma$ be an electoral equilibrium. By Theorem 5.1, each politician type $j$ mixes between two policies, $x_{j}^{*}$ and $x_{*, j}$, and the voter uses a cutoff $y^{*}$. Suppose there is a subsequence such that $y^{*}$ is bounded above, say $y^{*} \leqslant \bar{y}$. By Theorem 5.1, the equilibrium cutoff lies in the compact set $\left[\hat{x}_{1}, \bar{y}\right]$. Then the first order condition for the type 1 politician in (2) implies that $x_{*, 1} \rightarrow \infty$, and in particular, we have $\bar{y}<x_{*, 1}$ for large enough $\beta$, but this contradicts $x_{*, 1}+\hat{z} \leqslant$ $y^{*} \leqslant x_{n}^{*}+\hat{z}$. We conclude that $y^{*}$ diverges to infinity, which proves (i).

To prove (ii), suppose there is a type $j$, an $\varepsilon>0$, and a subsequence of office benefit levels such that $\hat{x}_{j}+\varepsilon \leqslant x_{j}^{*} \leqslant \frac{1}{\varepsilon}$. Going to a subsequence, we can assume $x_{j}^{*} \rightarrow \tilde{x}_{j}$ such that $\hat{x}_{j}<\tilde{x}_{j}<\infty$. Then for sufficiently large $\beta$, we have $\hat{x}_{j}<x_{j}^{*}$. For these parameters, the current gain to the type $j$ politician from choosing $\hat{x}_{j}$ instead of $x_{j}^{*}$ is non-positive, and thus we note that

$$
\left(F\left(y^{*}-\hat{x}_{j}\right)-F\left(y^{*}-x_{j}^{*}\right)\right)\left[w_{j}\left(\hat{x}_{j}\right)+\beta-V^{C}\right] \geqslant w_{j}\left(\hat{x}_{j}\right)-w_{j}\left(x_{j}^{*}\right) .
$$

That is, the current gains from choosing the ideal policy are offset by future losses. Since $y^{*} \rightarrow \infty$, the limit of

$$
\frac{F\left(y^{*}-x_{j}^{*}\right)-F\left(y^{*}-\tilde{x}_{j}-1\right)}{F\left(y^{*}-\hat{x}_{j}\right)-F\left(y^{*}-x_{j}^{*}\right)}
$$

as $\beta$ becomes large is indeterminate, and by L'Hôpital's rule, the limit is equal to

$$
\lim \frac{f\left(y^{*}-x_{j}^{*}\right)-f\left(y^{*}-\tilde{x}_{j}-1\right)}{f\left(y^{*}-\hat{x}_{j}\right)-f\left(y^{*}-x_{j}^{*}\right)}=\lim \frac{f\left(y^{*}-\tilde{x}_{j}-1\right)\left(\frac{f\left(y^{*}-x_{j}^{*}\right)}{f\left(y^{*}-\tilde{x}_{j}-1\right)}-1\right)}{f\left(y^{*}-x_{j}^{*}\right)\left(\frac{f\left(y^{*}-\hat{x}_{j}\right)}{f\left(y^{*}-x_{j}^{*}\right)}-1\right)}=\infty,
$$

where we use (C1) and (C2). Then, however, the future gain from choosing $\tilde{x}_{j}+1$ instead of $x_{j}^{*}$ strictly exceeds current losses, i.e.,

$$
\left(F\left(y^{*}-x_{j}^{*}\right)-F\left(y^{*}-\tilde{x}_{j}-1\right)\right)\left[w_{j}\left(\hat{x}_{j}\right)+\beta-V^{C}\right]>w_{j}\left(x_{j}^{*}\right)-w_{j}\left(\tilde{x}_{j}+1\right),(4)
$$

for high enough $\beta$. To be specific, let

$$
\begin{aligned}
A & =w_{j}\left(\hat{x}_{j}\right)+\beta-V^{C}, \\
B & =w_{j}\left(\hat{x}_{j}\right)-w_{j}\left(x_{j}^{*}\right), \text { and } \\
C & =w_{j}\left(x_{j}^{*}\right)-w_{j}\left(\tilde{x}_{j}+1\right),
\end{aligned}
$$


where $A$ is evaluated at sufficiently large $\beta$. Note that since $\hat{x}_{j}<\tilde{x}_{j}<\infty$, we have $\lim B>0$ and $\lim C<\infty$. We have noted that $\left(F\left(y^{*}-\hat{x}_{j}\right)-F\left(y^{*}-x_{j}^{*}\right)\right) A \geqslant B$ for sufficiently large $\beta$, and we have shown that as $\beta$ becomes large, we have

$$
\frac{F\left(y^{*}-x_{j}^{*}\right)-F\left(y^{*}-\tilde{x}-1\right)}{F\left(y^{*}-\hat{x}_{j}\right)-F\left(y^{*}-x_{j}^{*}\right)}>\frac{C}{B} .
$$

Combining these facts, we have

$$
\left(F\left(y^{*}-\hat{x}_{j}\right)-F\left(y^{*}-x_{j}^{*}\right)\right) A\left(\frac{F\left(y^{*}-x_{j}^{*}\right)-F\left(y^{*}-\tilde{x}-1\right)}{F\left(y^{*}-\hat{x}_{j}\right)-F\left(y^{*}-x_{j}^{*}\right)}\right)>B\left(\frac{C}{B}\right),
$$

which yields (4) for large $\beta$. This gives the type $j$ politician a profitable deviation from $x_{j}^{*}$, a contradiction. A similar argument holds for $x_{*, j}$, and (ii) follows.

Finally, to prove (iii), suppose that $x_{\ell-1}^{*}$ does not diverge to infinity. By (ii), there is a subsequence such that $x_{\ell-1}^{*} \rightarrow \hat{x}_{\ell-1}$. Now fix politician type $j \leqslant \ell-1$, and note that since equilibrium policy choices are ordered by type, we have $x_{j}^{*} \rightarrow \hat{x}_{j}$. Using the expression for Bayes rule, the posterior probability of type $j$ conditional on observing $y^{*}$ satisfies

$$
\mu_{T}\left(j \mid y^{*}\right)=\frac{p_{j} \sum_{x} f\left(y^{*}-x\right) \pi_{j}(x)}{\sum_{k} p_{k} \sum_{x} f\left(y^{*}-x\right) \pi_{k}(x)} \leqslant \frac{p_{j} f\left(y^{*}-x_{j}^{*}\right)}{\sum_{k \geqslant \ell} p_{k} \sum_{x} f\left(y^{*}-x\right) \pi_{k}(x)},
$$

where the inequality uses $y^{*} \rightarrow \infty$ and single-peakedness of $f(\cdot)$. Note that

$$
\sum_{k \geqslant \ell} p_{k} \sum_{x} f\left(y^{*}-x\right) \pi_{k}(x)=\sum_{k \geqslant \ell} p_{k}\left[f\left(y^{*}-x_{k}^{*}\right) \pi_{k}\left(x_{k}^{*}\right)+f\left(y^{*}-x_{*, k}\right) \pi_{k}\left(x_{*, k}\right)\right] .
$$

Dividing by $f\left(y^{*}-x_{j}^{*}\right)$, we obtain the expression

$$
\sum_{k \geqslant \ell} p_{k}\left[\frac{f\left(y^{*}-x_{k}^{*}\right)}{f\left(y^{*}-x_{j}^{*}\right)} \pi_{k}\left(x_{k}^{*}\right)+\frac{f\left(y^{*}-x_{*, k}\right)}{f\left(y^{*}-x_{j}^{*}\right)} \pi_{k}\left(x_{*, k}\right)\right] .
$$

By the MLRP, we have $\frac{f\left(y^{*}-x_{*, k}\right)}{f\left(y^{*}-x_{j}^{*}\right)} \rightarrow \infty$ for all $k \geqslant \ell$. Similarly, if $x_{k}^{*} \rightarrow \hat{x}_{k}$, then we have $\frac{f\left(y^{*}-x_{k}^{*}\right)}{f\left(y^{*}-x_{j}^{*}\right)} \rightarrow \infty$. By (ii), the remaining case is $x_{k}^{*} \rightarrow \infty$. Note that in this case, (C5) implies $w_{k}^{\prime}\left(x_{k}^{*}\right) \rightarrow-\infty$, and thus the first order condition in (2) implies that $f\left(y^{*}-x_{k}^{*}\right) \beta \rightarrow \infty$. The first order condition for type $j$ implies $f\left(y^{*}-x_{j}^{*}\right) \beta \rightarrow 0$, and we infer that $\frac{f\left(y^{*}-x_{k}^{*}\right)}{f\left(y^{*}-x_{j}^{*}\right)} \rightarrow \infty$. Thus, we have

$$
\mu_{T}\left(j \mid y^{*}\right) \leqslant \frac{p_{j}}{\sum_{k \geqslant \ell} p_{k} \sum_{x} \frac{f\left(y^{*}-x\right)}{f\left(y^{*}-x_{j}^{*}\right)} \pi_{k}(x)} \rightarrow 0 .
$$


We conclude that the voter's posterior beliefs conditional on $y^{*}$ place probability arbitrarily close to one on above average types $j \geqslant \ell$, contradicting the indifference condition in (3). Therefore, we have $x_{\ell-1}^{*} \rightarrow \infty$, and since policy choices are ordered by type, this implies that $x_{*, j} \rightarrow \infty$ for all $j \geqslant \ell$. This establishes (iii).

\section{Detailed literature review}

Closely related to our model is Fearon's (1999) model of "selection and sanctioning," with some important technical differences. First, Fearon assumes that there are just two types, that utility is quadratic, and that incentive constraints bind for only one type. Second, he assumes that a random shock is added directly to the voter's utility, and not to the underlying policy outcome; thus, Fearon's model cannot generally be interpreted as capturing an uncertain linkage between policy and observable variables, such as employment status, inflation, etc., on which voters might base their decisions. ${ }^{6}$ Third, Fearon argues (pp.86-90) that there are two candidates for a pure strategy equilibrium, but he does not prove existence of such an equilibrium. At issue is the possibility of non-convexities in the first-term office holder's objective function; this is exemplified in our Figure 4, and it arises in Fearon's model through non-concavity of the objective function in his equation (1). Because the derivation of his equation (7) relies on the first order condition in (2), it need not be that the desired policy choice $x_{b}=\sqrt{-2 k}$ is a global maximizer, even if it satisfies the second order condition. ${ }^{7}$ It is well-known that in non-convex games, existence of pure strategy equilibria is problematic, and this is true, in particular, of the electoral accountability model: it may be that none of the candidates for equilibrium identified by Fearon are, in fact, equilibria. In contrast, we impose sufficient structure on the model, in the form of (C0)-(C4), to establish existence of electoral equilibria and that mixing is limited to at most two policy choices for each politician type.

Chapter 3 of Besley (2006) presents a two-period, two-type model in which the incumbent politician observes the values of a binary state of the world and

\footnotetext{
${ }^{6}$ The distinction between utility and policy outcomes disappears when the voter is risk neutral, i.e., $u$ is affine linear, but the approaches are not equivalent in the general case, when the voter may be risk averse or risk loving.

${ }^{7}$ Because the distribution function $F$ is not convex, there may be multiple solutions to the office holder's first order condition, so that $x_{b}=\sqrt{-2 k}$ can be a local maximizer while failing to be a global maximizer. Note that this observation also applies in the case of Fearon's Figure 2.2a, as that case refers to a unique solution to the equilibrium equation (7), rather than the first order condition (2). Put differently, it may be that $x_{b}^{*}=\sqrt{-2 k^{*}}$ is a local but not global maximizer of the objective function $u(x)=W-(1-x)^{2}+\delta\left(1-F\left(x^{2}+k^{*}\right)\right) W$ given the unique solution $k^{*}$ to (7). Similar comments apply to Fearon's analysis of "pure sanctioning."
} 
preference shock, and then makes a binary policy choice. Closer to the model of our paper, Chapter 4 (coauthored with Smart) of the book investigates a twotype model in which an incumbent essentially chooses a level $x$ of shirking, and voters observe this with noise, $x+\varepsilon$. Besley and Smart assume, however, that the incumbent politician observes the policy shock $\varepsilon$ before her choice of $x$; in addition, the policy choice of the good type of politician is fixed exogenously. In these models, the politician's choice is either explicitly between two possible policies, or it reduces to a finite number of policies, so that equilibria in mixed strategies are assured to exist.

Chapter 4 of Persson and Tabellini (2000) contains a simplified, two-period model of symmetric learning, in which politicians are parameterized by a skill level that is unobserved by voters and politicians themselves. In this setting, voters and politicians update their beliefs symmetrically along the equilibrium path, and signaling cannot occur. Moreover, voters are assumed to be risk neutral. Ashworth (2005) considers a three-period model of symmetric learning that further differs from ours in that the skill level of a politician evolves over time according to a random walk. Although the model assumes three periods, the first-term office holder has private information about her ability only in the second and third terms, as her action in office are hidden from voters. Ashworth assumes that office benefit is small relative to the variance of policy outcomes in order to guarantee existence of equilibrium in pure strategies. Ashworth and Bueno de Mesquita (2008) use a variant of the model, one in which the voter has quadratic policy utility and a stochastic partisan preference, to establish existence and comparative statics of incumbency advantage.

Other work, including Barganza (2000) and Canes-Wrone et al. (2001), studies a two-type model in which politicians differ in ability. In the latter paper, the voter's desired policy depends on the realization of a state of the world, about which politicians are better informed. Politicians may have an incentive to pander to voters by knowingly choosing policies that are not in the voter's best interest. Maskin and Tirole (2004) study pandering in a two-type model in which politicians differ in preferences. Austen-Smith and Banks (1989) investigate the voter's ability to discipline politicians when all politicians have the same preferences, so that the model is one of pure moral hazard. In a two-period model of pure adverse selection, where politicians' policy choices are directly observed by voters, Duggan and Martinelli (2015) show that responsive democracy can arise due to the incentive of all politician types to "imitate" the median type. Theorem 6.1 in the current paper establishes that a form of this incentive holds for all above average types, delivering the responsiveness result even when policy choices are observed by voters with noise.

Due to difficult theoretical issues, the literature going beyond two periods is 
small. In an infinite-horizon version of our model, Banks and Sundaram (1993) find existence of (a continuum of) perfect Bayesian equilibria in which the voter uses a trigger strategy: if the observed policy outcome ever falls below a given level, then the voter resolves to replace the incumbent with a challenger, and the incumbent shirks for all remaining terms of office. The difficulty with such equilibria is that even if the incumbent is a good type with very high probability, there is a chance that the "trigger" is pulled, and then equilibrium strategies dictate that the otherwise attractive incumbent politician is replaced. Banks and Sundaram (1998) study the infinite-horizon model with a two-period term limit give an initial argument for existence of equilibrium. Duggan (2015) corrects an error in the argument of Banks and Sundaram and establishes limits on the possibility of responsive democracy in the infinite-horizon model: because voters cannot commit to replacing a politician after her first term of office, the voter's expected payoff from a first-term office holder is bounded strictly above by the expected utility from the ideal policy of the best politician type. Thus, the commitment problem of voters implies a qualitative difference between the two-period model and the infinite-horizon model with a two-period term limit.

\section{Conclusion}

The two-period model of elections provides a tractable setting for analysis of the interplay between short-term opportunistic incentives and long-term reelection incentives in determining politicians' behavior. We consider a natural, but technically challenging, environment in which voters are imperfectly informed about both the preferences and the actions of politicians, and we allow for an arbitrary finite set of politician types and general preferences. In line with the extant literature on electoral accountability, we assume that politicians and voters cannot commit to future actions, opening the scope for opportunistic behavior and creating potential difficulties for the success of democratic electoral mechanisms. We believe the two-period accountability model is a canonical framework in which to approach these issues, but despite this, foundational questions of equilibrium existence and responsiveness of policy to voter preferences have remained open. We address these questions by showing that office holder mix over at most two policy choices- "taking it easy" and "going for broke"—and establishing existence of electoral equilibrium. We then establish the possibility of responsive democracy: as politicians become more office motivated, the re-election standard used by voters becomes arbitrarily demanding, and the equilibrium level of expected effort in the first period becomes arbitrarily large. We conclude that incentives present in democratic elections have the potential to discipline the actions of elected rep- 
resentatives, mitigating the difficulties inherent in voters' sparse information and limited ability to sanction politicians.

\section{References}

Ashworth, S., 2005. Reputational dynamics and political careers. Journal of Law, Economics, and Organization 21, 441-466.

Ashworth, S., Bueno de Mesquita, E., 2008. Electoral selection, strategic challenger entry, and the incumbency advantage. Journal of Politics 70, 1006-1025.

Austen-Smith, D., Banks, J., 1989. Electoral accountability and incumbency. In: Peter Ordeshook (Ed.), Models of Strategic Choice in Politics. University of Michigan Press.

Bagnoli, M., Bergstrom, T., 2005. Log-concave probability and its applications. Economic Theory 26 (2), 445-469.

Banks, J., Sundaram, R., 1993. Adverse selection and moral hazard in a repeated election model. In: William Barnett et al. (Ed.), Political Economy: Institutions, Information, Competition, and Representation. Cambridge University Press.

Banks, J., Sundaram, R., 1998. Optimal retention in agency problems. Journal of Economic Theory 82, 293-323.

Barganza, J. C., 2000. Two roles for elections: Disciplining the incumbent and selecting a competent candidate. Public Choice 105, 165-193.

Barro, R., 1973. The control of politicians: An economic model. Public Choice 14, $19-42$.

Bernhardt, D., Duggan, J., Squintani, F., 2009. The case for responsible parties. American Political Science Review 103, 570-587.

Besley, T., 2006. Principled Agents? The Political Economy of Good Government. Oxford University Press.

Besley, T., Coate, S., 1997. An economic model of representative democracy. Quarterly Journal of Economics 112, 85-114.

Canes-Wrone, B., Herron, M., Shotts, K., 2001. Leadership and pandering: A theory of executive policymaking. American Journal of Political Science 45, $532-550$. 
Duggan, J., 2015. Term limits and policy responsiveness in dynamic elections. Unpublished paper.

Duggan, J., Martinelli, C., 2015. The political economy of dynamic elections: Accountability, commitment, and responsiveness. Unpublished paper.

Fearon, J., 1999. Electoral accountability and the control of politicians: Selecting good types versus sanctioning poor performance, in Bernard Manin, Adam Przeworski and Susan Stokes (eds.). In: Przeworski, A., Stokes, S., Manin, B. (Eds.), Democracy, Accountability, and Representation. Cambridge University Press.

Ferejohn, J., 1986. Incumbent performance and electoral control. Public Choice $50,5-25$.

Maskin, E., Tirole, J., 2004. The politician and the judge: Accountability in government. American Economic Review 94, 1034-1054.

Osborne, M., Slivinski, A., 1996. A model of competition with citizen candidates. Quarterly Journal of Economics 111, 65-96.

Persson, T., Tabellini, G., 2000. Political Economics: Explaining Economic Policy. MIT Press.

Roemer, J., 1997. Political-economic equilibrium when parties represent constituents: The unidimensional case. Social Choice and Welfare 14, 479-502. 